\title{
"I've Weathered Really Horrible Storms Long Before This...": The Experiences of Parents Caring for Children with Hematological and Oncological Conditions during the Early Months of the COVID-19 Pandemic in the U.S.
}

\author{
Dara M. Steinberg ${ }^{1,2} \cdot$ Jane A. Andresen ${ }^{3} \cdot$ Daniel A. Pahl ${ }^{4} \cdot$ Maureen Licursi $^{5} \cdot$ Susan L. Rosenthal ${ }^{2,3}$
}

Accepted: 24 December 2020 / Published online: 25 January 2021

(c) The Author(s), under exclusive licence to Springer Science+Business Media, LLC part of Springer Nature 2021

\begin{abstract}
The societal impact of COVID-19 is vast, thus it is imperative to understand how vulnerable groups, such as children with chronic medical conditions are affected. This understanding can prepare psychologists and other healthcare providers to meet their current and future needs. A convenience sample of 11 parents of children with hematological/oncological conditions was recruited. They participated in semi-structured interviews on the effect of the COVID-19 pandemic on their children. Qualitative analysis identified common themes. Parental responses focused on the pandemic's impact on children's general daily life and healthcare. Themes of caution, uncertainty, adaptation, and the role of the healthcare providers and early medical experiences emerged. Concerns about vulnerability, changes in routine, the importance of virtual connections, and the pivotal role of providers have implications for children with and without medical conditions. The adaptation and resilience of the families provide a sense of hope in an uncertain time.
\end{abstract}

Keywords Pediatrics $\cdot$ Hematology/oncology/bone marrow transplant $\cdot$ Nurse practitioners $\cdot$ Resilience $\cdot$ Telehealth

Dara M. Steinberg

dms2207@cumc.colulmbia.edu

Jane A. Andresen

ja3337@cumc.columbia.edu

Daniel A. Pahl

dap2150@cumc.columbia.edu

Maureen Licursi

mal9068@nyp.org

Susan L. Rosenthal

slr2154@columbia.edu

1 Department of Pediatrics, Division of Hematology, Oncology \& Stem Cell Transplantation, Columbia University Irving Medical Center, 161 Fort Washington Avenue-IP 7, New York, NY 10032, USA

2 Department of Psychiatry, Division of Child \& Adolescent Psychiatry, Columbia University Irving Medical Center, 161 Fort Washington Avenue-IP 7, New York, NY 10032, USA

3 Department of Pediatrics, Columbia University Irving Medical Center, New York, USA

4 Vagelos College of Physicians and Surgeons, Columbia University Irving Medical Center, New York, USA

5 Morgan Stanley Children's Hospital, NewYork-Presbyterian Hospital, New York, USA

\section{Introduction}

The novel coronavirus 2019 (COVID-19) has had a significant effect on almost every aspect of life in the United States (U.S.) including school closures, stay-at-home orders, and widespread unemployment (Galea \& Abdalla, 2020; Jacobson et al., 2020; Xiang, Zhang, \& Kuwahara, 2020). Although children appear to be less medically impacted by COVID-19 (Centers for Disease Control and Prevention, 2020; Panahi, Amiri, \& Pouy, 2020), those with immunocompromising or chronic health conditions are in unique and potentially vulnerable circumstances compared to children without chronic conditions (Feldstein et al., 2020; Zachariah et al., 2020). In addition to being at higher risk for infection and complications, children with chronic health conditions may also be affected by health service changes. Specifically, the pandemic has limited access to routine in-person care for chronic conditions, as medical centers have tried to reduce the risk of person-to-person transmission of COVID-19 (Feldstein et al., 2020; Zachariah et al., 2020). For instance, one retrospective analysis of 174 pediatric hematology, oncology, and hematopoietic stem cell transplant patients 
(some from the same institution as this study) found that nearly two-thirds of patients with cancer experienced treatment delays due to COVID-19 mostly due to decisions to defer planned treatment (Gampel et al., 2020). The aim of the current study was to explore parents' descriptions of their experiences caring for their children with hematological/oncological conditions during the early phases of the pandemic, to help gain an understanding of what the implications of COVID-19 have been for their daily lives as well as healthcare management.

\section{Method}

A convenience sample was recruited from the Pediatric Hematology, Oncology and Stem Cell Transplantation Center at Columbia University Irving Medical Center, New York, New York. Individuals were eligible for participation if: they were the primary caregiver (hereby referred to as parent) of a child actively in care for a malignant or nonmalignant hematological/oncological condition (if there was more than one primary caregiver identified it was at the parents' discretion who participated); their child had been diagnosed prior to age 18; they spoke English. Parents were recruited during previously scheduled clinical care visits (in-person and telemedicine) until theoretical saturation was reached. They participated in a semi-structured qualitative interview. Clinical data were abstracted from their children's medical records. Interviews took place from the end of April to the beginning of June 2020. During the study period the "New York on Pause" executive order had been signed and all non-essential personnel were told to stay home (New York State Government, 2020). School was being held virtually and New York City was approaching Phase I of reopening (occurred 2 days before the final data collection point). The study was approved by the Institutional Review Board (IRB) of Columbia University Irving Medical Center.

\section{Interview}

The semi-structured interview focused on the effect that COVID-19 was having on the daily lives of the parents' children and on their children's healthcare. At the time of study design there were no standardized measures to capture the impact of COVID-19, thus the interview was created by the authors whose combined expertise include qualitative research, pediatric psychology, and medicine. Two of the authors were actively engaged in patient care during the time of interview creation. As themes emerged during initial interviews, the interview was modified. Interviews were conducted by one of the authors (JAA) a clinical research coordinator. They were done telephonically, recorded and transcribed. Interviews ranged from 15.92 to 43.0 min with a mean of $28.45 \pm 7.99 \mathrm{~min}$.

\section{Clinical Data}

Extracted data included: child's medical diagnosis; age at diagnosis; current age; gender; insurance type.

\section{Data Analysis}

Qualitative data were coded using framework theory (Gale, Heath, Cameron, Rashid, \& Redwood, 2013). Two independent coders (DMS, DAP) read and coded the transcripts. A third coder (JAA) met with DMS and DAP to establish consensus. After initial coding and consensus, the entire study team met, and coding was refined. Clinical data were analyzed using measures of central tendency (i.e., mean, median, percentage).

\section{Results}

\section{Participants}

In total 18 parents were approached for the study; 2 declined, 5 could not be reached after they initially expressed interest in the study, and 11 participated. Reasons for not participating were not provided. The majority $(n=10,91 \%)$ were mothers, and one was a male guardian. The children were between the ages of 2 to $18(M$ age $=8.73+5.33 ; M d$ age $=9.00 ; 54.5 \%$ males $)$ with non-malignant $(n=9,81.8 \%)$ and malignant ( $n=2,18 \%)$ conditions (Table 1). The most common condition was sickle cell disease $(n=6,63.6 \%)$; other conditions included: vascular malformation; immune thrombocytopenia; acute lymphoblastic leukemia; solid tumor. The majority $(n=8,72.7 \%)$ of the children were diagnosed prenatally or at birth. Most $(n=8,72.7 \%)$ had private insurance.

\section{Primary Themes}

Parents' responses focused on the impact of the COVID-19 pandemic on two primary areas: general daily life and their children's medical care. Within these areas the main themes that emerged were caution, uncertainty, adaptation, and the role of healthcare providers and early medical experiences in navigating change (Table 2).

\section{Caution in Daily Life}

Parents reported that prior to the start of the pandemic their daily lives were filled with routines and a sense of normalcy. As COVID-19 emerged in the U.S. they reported 
Table 1 Characteristics of participants

\begin{tabular}{llr}
\hline Variable & $n$ & $\% *$ \\
\hline Child & $\mathrm{M} \pm \mathrm{SD}$ & \\
Age & $8.73 \pm 5.33$ & \\
Gender & & 45.5 \\
Female & 5 & 54.5 \\
Male & 6 & \\
Race & & 30.0 \\
White & 3 & 50.0 \\
Black or African American & 5 & 1.0 \\
Asian & 1 & 1.0 \\
Two or more races & 1 & 72.7 \\
Ethnicity & & 27.3 \\
Latino or Hispanic & 8 & 18.2 \\
Non-Latino or non-Hispanic & 3 & 18.8 \\
Medical diagnosis & & \\
Malignant condition & 2 & 27.3 \\
$\quad$ Non-malignant condition & 9 & 72.7 \\
Insurance & & \\
Public & & \\
Private & & \\
Caregiver gender & & \\
Female & & \\
Male & & \\
\hline & &
\end{tabular}

*Percent of responses given

that their children's vulnerability due to their medical conditions led them to make changes in their routines to protect them. All parents reported following the guidelines set forth by regulatory bodies. This included: family members remaining in the home (e.g., virtual school, working from home if not essential workers), limiting exposure to those not living in the home, and wearing masks. One participant described initially taking extra precautions: “...I put us on house arrest at the end of February. I pulled [my child] and my older one from school before anything shut down in New York...I wasn't really willing to wait until I understood just simply because [my child] is so fragile, it's not worth it." Yet, this same parent reported relaxing rules as the pandemic continued, allowing her child to play with neighbors stating, "there's no social distancing children" (4-year-old child, non-malignant condition). Another parent reported allowing her children to play with cousins, explaining "Because this is really hard for the kids. They just can't take it anymore" (9-year-old child, non-malignant condition). Yet, other parents indicated that they would continue to take precautions even when the area reopened. For instance, one reported "...we definitely will be distancing ourselves. We have already agreed to keep ourselves under quarantine. It's going to be a while before we're actually living a normal life" (9-year-old child, malignant condition).

\section{Uncertainty and Their Adaptation in Daily Life}

Parents reported an overall sense of uncertainty related to COVID-19 and what it would mean for their children's daily lives. This level of overall uncertainty is illustrated by the statement, "It was scary because it was very unknown. It's almost like you're hearing, the big tornado's coming, and it's coming right at you, and you don't have anywhere to go... It's like you're trying to brace yourself and not knowing what's going to happen" (11-year-old child, non-malignant condition).

Parents reported changes in terms of their children's daily routines, moods, and overall developmental progression. In terms of their daily routines, parents reported it was difficult to make all extracurricular activities educational, and that they had shifted their views on activities, including prior limits to screen time. Parents reported that technology allowed their children to connect with others, for school and play, e.g., "Well, they do have Zoom calls with their friends" (16-year-old child, non-malignant condition); “...They have little meetings about math. They share the writing. I think he always looks forward to those meetings. That way, he will see his friends from school. The mood is better when he has those meetings to look forward to" (9-year-old child, nonmalignant condition). They reported that school provided some sense of normalcy. Parents described enjoying time spent together as a family, e.g., "But overall, the experience has been, I think, a bit of a gift because my two boys now are inseparable. They have all these imaginary games they play" (4-year-old child, non-malignant condition). In terms of mood, most of the parents reported in general their children remained happy and were having fun, e.g., "they're still kids you know" (4-year-old child, non-malignant condition). A few reported their children were moodier. One mother spoke about feeling concerned about how missing a medical milestone might affect her child's mood in the future, stating “...I feel like sometimes I'm trampling on the possibility for joy ..." (18-year-old child, malignant condition). In terms of overall development, a few also reported that their children were not able to adapt to changes in service delivery, specifically occupational therapy, speech therapy, and physical therapy, stating that they noted their children regressing.

Parents reported their children generally (with the exception of the youngest children 2- to 4-year-old) seemed to have a level of understanding of what COVID-19 meant, and why it was important to remain safe. They reported openly sharing information with their children about what was occurring. Overall, they denied that their conversations about COVID-19 differed between their children with and without medical conditions. 
Table 2 Themes

\begin{tabular}{|c|c|c|}
\hline & Themes & Additional quotes \\
\hline \multirow[t]{4}{*}{ General daily life } & Caution & $\begin{array}{l}\text { "Everything is crazy at this point with making decisions. I think that I just try to, when I'm making any } \\
\text { decision, even just besides [about my child's] health, with just overall just thinking about her and how } \\
\text { I'm going to do certain things, I'm always thinking safety." (6-year-old child, non-malignant) }\end{array}$ \\
\hline & Uncertainty & $\begin{array}{l}\text { "I, myself, I work in the healthcare field and I had requested not to work directly with the COVID unit } \\
\text { because when he was first diagnosed, he did have two viral illnesses. They said that could have poten- } \\
\text { tially caused him to go down to that critically low level. Not knowing exactly what COVID does, that } \\
\text { also, yeah, really scared me." (2-year-old child, non-malignant) }\end{array}$ \\
\hline & Adaptation & $\begin{array}{l}\text { "... We were coming out of the hospital initially... We would have been } 100 \text { days in isolation anyway. } \\
\text { You know, we entered into this already relatively isolated." (18-year-old child, malignant) }\end{array}$ \\
\hline & $\begin{array}{l}\text { Role of HCP } \\
\text { \& early } \\
\text { medical } \\
\text { experiences }\end{array}$ & $\begin{array}{l}\text { “...if I have any questions, I'll ask her [nurse practitioner]. If I have any concerns, I'll ask her, what } \\
\text { should I do or not do with [my child]? She'll let me know. I do what she tells me usually... something } \\
\text { that I want to ask... how healthy it is for me to be able to take her out for a walk?” (2-year-old child, } \\
\text { non-malignant) }\end{array}$ \\
\hline \multirow[t]{4}{*}{ Children's medical care } & Caution & $\begin{array}{l}\text { "They were trying to change the dosage of one of the meds that he's taking... But it's going to require } \\
\text { a lot of blood work...It's not that we can't get it done, but they don't want to expose him too much." } \\
\text { (12-year-old child, non-malignant condition) }\end{array}$ \\
\hline & Uncertainty & $\begin{array}{l}\text { "We have also... stocked up on all of her medications in the event that, for whatever reason, we can't } \\
\text { access it." (6-year-old child, non-malignant) }\end{array}$ \\
\hline & Adaptation & $\begin{array}{l}\text { "But then she also has a deeper understanding of the risks, and what that risk could bring to her life. } \\
\text { She has memories of hospitalization that she doesn't like, so that helps. Also, silver linings, I have an } \\
\text { easier time, I think, than most parents getting her to be safe because she has a very real idea of what } \\
\text { she's trying to avoid." (6-year-old child, non-malignant) }\end{array}$ \\
\hline & $\begin{array}{l}\text { Role of HCP } \\
\text { \& early } \\
\text { medical } \\
\text { experiences }\end{array}$ & $\begin{array}{l}\text { "Actually for coronavirus, I waited [for medical care] because...I'll call [his nurse practitioner] and } \\
\text { I'll [ask her]... how important this [medical care is] because I don't want to expose him." (9-year- } \\
\text { old child, non-malignant) }\end{array}$ \\
\hline
\end{tabular}

\section{Medical Care and Caution}

When it came to their children's medical care, parents described exercising caution to the extent that they were able, to reduce risk of COVID-19 infection. One parent described the hospital as "ground zero" (18-yearold child, malignant condition). When possible parents sought care outside the medical center (e.g., local laboratories for bloodwork, identified local providers and emergency care options, appreciating telemedicine visits) or postponed non-emergent care (e.g., changing dosing of medication). One parent reported feeling reassured after her son needed to go to the hospital when current treatment was no longer effective, stating "...there is actually no need for me to have fear ..." (9-year-old child, malignant condition)." In addition to making decisions in the context of their children's medical care with additional considerations given to COVID-19, one parent reported explaining more about her child's medical condition to her. She stated "I think she has a greater understanding of what it means to have sickle cell than she did a few months ago...she has to understand that she is... in that at-risk class..." (6-year-old child, non-malignant condition).

\section{Medical Care: Uncertainty and Adaptation}

Parents also reported experiencing initial uncertainty about how they would manage their children's medical needs during the pandemic. One mother stated that her child's medical care is "...part of our lives, but it's not a thing that we focus on in any way, shape, or form ...I think corona has brought all of it back up to the forefront of our lives, which has been a bit more stressful" (6-year-old child, non-malignant condition). Parents reported that at the start of the pandemic they were uncertain about access to medication and medical supplies. They worked with their medical providers to identify local providers including laboratories, pediatricians, and emergency providers to avoid traveling to the medical center. They reported feeling reassured as they executed new healthcare plans and saw that these experiences could be positive. 


\section{The Pivotal Role of the Healthcare Provider and Early Medical Experiences}

Parents reported that prior to the COVID-19 pandemic they had longstanding relationships and high levels of trust in their medical providers. The extent of their trust is illustrated by the fact that when asked about medical decisionmaking, the majority reported they did not make decisions, but instead followed the recommendations of their providers. One parent stated, "Where his doctors are concerned, definitely we do listen to everything that they tell us.... at the end of the day, they are the ones that are helping us to keep him alive, so we would follow all their instructions" (9-year-old child, malignant condition). During the COVID19 pandemic they relied on and adapted these relationships to help them navigate the changes they were experiencing. Many reported increased communication with their nurse practitioner, seeking guidance on both daily life (e.g., should their children go outside) and medical care (e.g., getting blood draws, local providers, medications). One reported: "I think I've talked to [her nurse practitioner] by phone more often than I usually do. It's been such a long time [with her medical condition] that everything is routine at this point. It's become a bit more routine in the past few weeks" (6-year-old child, non-malignant condition). Parents reported that they appreciated being able to maintain the relationship with their nurse practitioner and other providers, despite decreased frequency of in-person medical care. The majority were appreciative of the option for telemedicine and described it as a means of continuing communication and connection while also limiting potential exposures to COVID-19. One participant spoke about the limitations of telemedicine (i.e., not being able to have her child physically examined) while another reported a sense of being disconnected, although feeling her child was medically taken care of: "I don't want to say like, oh, they're neglecting him. They're not because they would know, according to his blood work, what needs to be done...But I just feel like everything is so, I guess distanced..." (2-year-old child, non-malignant condition).

In addition to the guidance from their healthcare providers, parents reported that they and their children were wellequipped to deal with uncertainty and adapt to the pandemic given their history of managing their medical conditions, many since birth. One stated: "The medical team and I have been [managing] his care for years now. We're prepped uniquely for this type of situation because generally speaking, most parents, who have medically complex kids tend to become experts in their child's disease and can usually navigate most things themselves. They can nurse their kid. I'm fortunate in that I've weathered really horrible storms long before this that I really know how to keep him safe during this" (4-year-old child, non-malignant condition). Another participant stated "We're doing this for over three years... We understand that there are things you just have to do to get through the cycle, whatever that cycle is currently. I mean, we mask up. We use Purell. We try to be as cautious as possible, you know, and try not to be guided necessarily by fear" (18-year-old child, malignant condition). They reported their children were familiar with taking precautions, including wearing masks and staying away from others due to their medical histories. For instance, one parent stated, "We were already in quarantine. The last time we actually spent time with any family member... was for Christmas week" (9-year-old child, malignant condition).

\section{Discussion}

The COVID-19 pandemic is a unique experience that has affected innumerable aspects of daily life for children, adolescents, and their parents. The potential impact that the pandemic may have on children's physical and emotional health and development highlights the importance of gaining an in-depth understanding of family's experiences during this time. Children with chronic medical conditions have pre-existing challenges that make them an especially important group to consider. This study highlights that these children and their families experienced effects of the pandemic both in their daily lives and specifically in relation to their medical care. They spoke about exercising caution driven in part by feelings of vulnerability, uncertainty, and ultimately adaptation. Their adaption was considerably bolstered by the guidance and support of their healthcare providers, as well as the sense of resilience they had ingrained in them from years of coping with/caring for chronic illnesses. These themes have important implications for children with and without chronic conditions and can better help the healthcare community respond to current and emerging needs of children due to the pandemic. Psychologists in medical settings can play a key role through psychoeducation to families and multidisciplinary team members, and providing screening and intervention.

While it was not the main focus of parents' responses, some did report lower mood amongst their children, and there is emerging research on the impact of the pandemic and quarantine on mental health. As the pandemic continues, mood may be a growing area of concern as early research has shown increases in depression, anxiety, and stress during the early stages of the pandemic (Talevi et al., 2020). Research from China suggests psychological implications including depression and post-traumatic stress disorder associated with direct exposure to COVID19 , more intense exposure to media, and perceived negative impact on livelihood (Guo, Feng, Wang, \& van 
IJzendoorn, 2020). Additionally, previous pandemics and mass disasters have demonstrated significant mental health impacts (Brooks et al., 2020). For example, longer durations of quarantine in the SARS epidemic were shown to be associated with a higher prevalence of post-traumatic stress disorder (PTSD) (Hawryluck et al., 2004). Thus, continued assessment of children's adjustment and mood will be important to ensure early intervention if needed.

In terms of their children's daily lives, parents highlighted changes in routines and access to supportive services. The use of electronics featured heavily in children's lives during the pandemic, from virtual school, to connecting with peers, to telemedicine. Parents who reported regulating time on electronics pre-pandemic, noted the importance of electronics in keeping their children connected to others. This has important implications for a child with medical conditions, as social support and connectedness has been demonstrated to be protective in medically vulnerable populations (e.g., Ehman, Schepers, \& Phipps, 2019; Sharp et al., 2015). Continuing virtual forms of education, activities, and socialization even as pandemic restrictions lift, could be beneficial to children with chronic conditions. Yet, remaining aware of recommendations regarding screen time and weighing benefits of screen use versus potential consequences such as decreased physical activity and disrupted sleep is important.

Additionally, parents reported a great deal of benefit from telemedicine visits, as this allowed them to remain connected to their children's medical teams without incurring extra risk of COVID-19 infection. Nationally healthcare systems have significantly adapted their health service delivery modalities across medical specialties to integrate telemedicine (Barney, Buckelew, Mesheriakova, \& Raymond-Flesch, 2020; Mann, Chen, Chunara, Testa, $\&$ Nov, 2020; Steinberg et al., 2020). Continuing to offer this as an adjunct to in-person services has the potential to both mitigate potential risk in pandemic-like situations, as well as to keep patients more engaged in their medical care long-term. Parents in this study reported a high level of trust and connection to their medical providers, specifically their nurse practitioner, which has been demonstrated in other studies highlighting the important role the patient-provider relationship plays in adherence (e.g., Crosby et al., 2009; Schoenthaler, Rosenthal, Butler, \& Jacobowitz, 2018). Telemedicine provides access to care with less disruption to work/school schedules, incurring travel costs, or loss of income due to time-off. Long-term this has the potential to improve adherence to treatment regimens and ultimately health outcomes. Yet, the limitations of telemedicine that parents described are important to acknowledge and assess for, including physical examinations as well as occupational and physical therapy.
While the families in this study likely have more frequent contact with the medical system, healthcare providers can also play a similar role for families whose children do not have chronic medical conditions. Annual check-ups and perhaps more frequent check-ins, as the pandemic continues, can be a key time to answer questions, address uncertainties and assess for areas that have emerged as concerns during the pandemic, such as access to healthcare, mood, and overall development.

Parents also had a significant focus on the effect of the pandemic on caring for their children's medical needs. Initial concerns included keeping their children safe due to vulnerabilities related to their conditions, as well as ensuring continued access to medications and care. Their report of vulnerability is consistent with international research, in which pediatric patients and parents reported feeling as if their children were more at risk for complications related to COVID-19. In an Italian study children also indicated feeling the burden of their parents' concern for them (Casanova et al., 2020; Darlington et al., 2020; Mirlashi et al., 2020). Similarly, in the current study, parents expressed concern and the need for caution when accessing medical services, which suggests the need for continued public education on practices healthcare systems have taken to ensure safety from disease transmission. Parents of children with oncological conditions in England also reported feeling as if the hospital were no longer a safe place for their children due to COVID-19 (Darlington et al., 2020). This shifting perception of the hospital and healthcare system is especially important given growing concern about children missing key vaccinations and others avoiding care until illness have progressed. Thus, it is important to ensure parents are aware of the American Academy of Pediatrics (2020) recommendations to follow set standards of well-child care as outlined by the Bright Futures Guidelines for Health Supervision of Infants, Children and Adolescents. Finally, parents and children adapted during the pandemic and found benefit in changes in some of their previous healthcare practices, such as going to local laboratories for bloodwork and the use of telemedicine.

The ability of the parents and their children to adapt to the changes they were experiencing evidences their resilience. Despite the additional challenges they faced navigating the pandemic with chronic health conditions and complex medical needs, their previous experiences dealing with hardship appeared to be protective. This is consistent with literature that has shown that children with chronic conditions (e.g., Picoraro, Womer, Kazak, \& Feudtner, 2014; Tillery, Howard Sharp, Okado, Long, \& Phipps, 2016) and their parents (e.g., Phipps et al., 2015; Reader et al., 2020) exhibit resilience and post-traumatic growth compared to peers. The idea of adaptation is also consistent with reports from other countries (Mirlashari, Ebrahimpour, \& Salisu, 2020). Many of 
the skills the families in this study had to develop and use to manage the uncertainty, change, and challenges associated with their children's medical conditions were able to be translated to managing these same aspects of the COVID-19 pandemic, including the connections with their healthcare providers. The majority of parents in this study had been managing their children's medical conditions for their entire lives, thus they appeared to be competent and able to manage the new challenges they experienced with COVID-19. As the pandemic continues and its implications continue to emerge, resilience-building can help children and their parents prepare for the days to come and manage future challenges that may emerge over the course of their lifetime. Additionally, similar to the children described in this study whose parents reported they knew about COVID-19, encouraging parents to have continued open and developmentally appropriate conversations with their children about COVID-19 and its implications is important. Honest communication as well as helping children orient toward ways they can be in control (e.g., practicing safety measures) can promote positive mental health during an uncertain time (Weaver \& Weiner, 2020).

While this study provides an initial understanding of the experiences of parents and children with chronic illness during the early months of the COVID-19 pandemic in the United States, there are important limitations to address. The study occurred during a period filled with great uncertainty and many daily changes, thus it may serve more as a snapshot of experiences as opposed to the overall experience these children and their parents had. Additionally, the children were all recruited from the same medical center, and many had the same providers, potentially limiting generalizability across medical settings. The sample size was small, limiting overall generalizability. Additionally, the majority of the children had non-malignant conditions, thus there are likely aspects of caring for children with malignant conditions that were not captured in this study. Those participating may also be unique and overall more adaptable and compliant, as they were recruited during medical care, thus were continuing to engage with their medical teams despite the pandemic. There is likely an important group of children and parents with chronic medical conditions who have not maintained such connections during this time and whose experiences are also important to understand. Due to the dynamic nature of the pandemic, the interview was developed during a time when the affects and implications of the pandemic were not yet understood. While the semi-structured nature of the interview allowed for adjustments based on the lived experiences of the participants, there were many areas that we are now beginning to understand the importance of, such as mental health of children, parents, and people in general, virtual school, economic stressors, and the role of racial/ ethnic disparities, that were not captured.
Future research will be important in continuing to understand the impact the COVID-19 pandemic is having on children with chronic medical conditions and the role psychologists can play in promoting overall positive outcomes. Serial evaluations can help understand long-term effects, as well as combining this with physiological data on overall medical status. Expansion to other medical conditions, as well as different areas of the country with other healthcare systems could also be important. Coupling the current study with the perspectives of the children themselves as well as their healthcare providers may also provide important insight into the effects that the pandemic is having. This could help better prepare the medical system to assess and address potential long-term implications of the pandemic.

Acknowledgements We would like to thank the parents who spoke with us during this uncertain time.

Funding Dara M. Steinberg's position is supported by The Valerie Fund.

\section{Compliance with Ethical Standards}

Conflict of interest The authors report no conflicts of interest.

Human and Animal Rights and Informed consent The study was approved by the Insitutional Review Board of Columbia University Irving Medical Center and informed consent was obtained from all participants.

\section{References}

American Academy of Pediatrics. (2020). Guidance on providing pediatric well-care during COVID-19. Retrieved from https://services. aap.org/en/pages/2019-novel-coronavirus-covid-19-4infections/ clinical-guidance/guidance-on-providing-pediatric-well-careduring-covid-19/.

Barney, A., Buckelew, S., Mesheriakova, V., \& Raymond-Flesch, M. (2020). The COVID-19 pandemic and rapid implementation of adolescent and young adult telemedicine: Challenges and opportunities for innovation. Journal of Adolescent Health, 67, 164-171. https://doi.org/10.1016/j.jadohealth.2020.05.006.

Brooks, S. K., Webster, R. K., Smith, L. E., Woodland, L., Wessely, S., Greenberg, N., \& Rubin, G. J. (2020). The psychological impact of quarantine and how to reduce it: Rapid review of the evidence. Lancet, 395, 912-920. https://doi.org/10.1016/s0140 $-6736(20) 30460-8$.

Casanova, M., Pagani Bagliacca, E., Silva, M., Patriarca, C., Veneroni, L., Clerici, C. A., ... Ferrari, A. (2020). How young patients with cancer perceive the COVID-19 (coronavirus) epidemic in Milan, Italy: Is there room for other fears? Pediatric Blood \& Cancer, 67, e28318. https://doi.org/10.1002/pbc.28318.

Center for Disease Control and Prevention. (2020). Information for pediatric healthcare providers. Retrieved from https://www.cdc. gov/coronavirus/2019-ncov/hcp/pediatric-hcp.html.

Crosby, L. E., Modi, A. C., Lemanek, K. L., Guilfoyle, S. M., Kalinyak, K. A., \& Mitchell, M. J. (2009). Perceived barriers to clinic 
appointments for adolescents with sickle cell disease. Journal of Pediatric Hematology and Oncology, 31, 571-576. https://doi. org/10.1097/MPH.0b013e3181acd889.

Darlington, A. E., Morgan, J. E., Wagland, R., Sodergren, S. C., Culliford, D., Gamble, A., \& Phillips, B. (2020). COVID-19 and children with cancer: Parents' experiences, anxieties and support needs. Pediatric Blood \& Cancer. https://doi.org/10.1002/ pbc. 28790

Ehman, A. C., Schepers, S. A., \& Phipps, S. (2019). The effect of optimism and connectedness on psychological adjustment of children with cancer and comparison peers. Journal of Developmental \& Behavioral Pediatrics, 40, 208-216. https://doi.org/10.1097/ dbp.0000000000000641.

Feldstein, L. R., Rose, E. B., Horwitz, S. M., Collins, J. P., Newhams, M. M., Son, M. B. F., ... the CDC COVID-19 Response Team. (2020). Multisystem inflammatory syndrome in U.S. children and adolescents. New England Journal of Medicine, 383, 334-346. https://doi.org/10.1056/NEJMoa2021680.

Gale, N. K., Heath, G., Cameron, E., Rashid, S., \& Redwood, S. (2013). Using the framework method for the analysis of qualitative data in multi-disciplinary health research. BMC Medical Research Methodology, 13, 117. https://doi.org/10.1186/1471-2288-13-117.

Galea, S., \& Abdalla, S. M. (2020). COVID-19 pandemic, unemployment, and civil unrest: Underlying deep racial and socioeconomic divides. Journal of the American Medical Association. https://doi. org/10.1001/jama.2020.11132.

Gampel, B., Troullioud Lucas, A. G., Broglie, L., Gartrell-Corrado, R. D., Lee, M. T., Levine, J., ... Roberts, S. S. (2020). COVID19 disease in New York City pediatric hematology and oncology patients. Pediatric Blood \& Cancer, e28420. https://doi. org/10.1002/pbc.28420.

Guo, J., Feng, X. L., Wang, X. H., \& van IJzendoorn, M. H. (2020). Coping with COVID-19: Exposure to COVID-19 and negative impact on livelihood predict elevated mental health problems in Chinese adults. International Journal of Environmental Research and Public Health. https://doi.org/10.3390/ijerph17113857.

Hawryluck, L., Gold, W. L., Robinson, S., Pogorski, S., Galea, S., \& Styra, R. (2004). SARS control and psychological effects of quarantine, Toronto, Canada. Emerging Infectious Diseases, 10, 1206-1212. https://doi.org/10.3201/eid1007.030703.

Howard Sharp, K. M., Willard, V. W., Okado, Y., Tillery, R., Barnes, S., Long, A., \& Phipps, S. (2015). Profiles of connectedness: Processes of resilience and growth in children with cancer. Journal of Pediatric Psychology, 40, 904-913. https://doi.org/10.1093/ jpepsy/jsv036.

Jacobson, N. C., Lekkas, D., Price, G., Heinz, M. V., Song, M., O'Malley, A. J., \& Barr, P. J. (2020). Flattening the mental health curve: COVID-19 stay-at-home orders are associated with alterations in mental health search behavior in the United States. Journal of Medical Internet Research Mental Health, 7, e19347. https ://doi.org/10.2196/19347.

Mann, D. M., Chen, J., Chunara, R., Testa, P. A., \& Nov, O. (2020). COVID-19 transforms health care through telemedicine: Evidence from the field. Journal of the American Medicial Informatics Association, 27, 1132-1135. https://doi.org/10.1093/jamia locaa072.

Mirlashari, J., Ebrahimpour, F., \& Salisu, W. J. (2020). War on two fronts: Experience of children with cancer and their family during COVID-19 pandemic in Iran. Journal of pediatric nursing, 57, 25-31. https://doi.org/10.1016/j.pedn.2020.10.024.
New York State Government. (2020). Governor Cuomo issues guidance on essential services under the 'New York State on PAUSE' Executive Order. Retrieved from https://www.governor.ny.gov/ news/governor-cuomo-issues-guidance-essential-services-under -new-york-state-pause-executive-order.

Panahi, L., Amiri, M., \& Pouy, S. (2020). Clinical characteristics of COVID-19 infection in newborns and pediatrics: A systematic review. Archives of Academic Emergency Medicine, 8, e50.

Phipps, S., Long, A., Willard, V. W., Okado, Y., Hudson, M., Huang, Q., ... Noll, R. (2015). Parents of children with cancer: At-risk or resilient? Journal of Pediatric Psychology, 40, 914-925. https:// doi.org/10.1093/jpepsy/jsv047.

Picoraro, J. A., Womer, J. W., Kazak, A. E., \& Feudtner, C. (2014). Posttraumatic growth in parents and pediatric patients. Journal of Palliative Medicine, 17, 209-218. https://doi.org/10.1089/ jpm.2013.0280.

Reader, S. K., Pantaleao, A., Keeler, C. N., Ruppe, N. M., Kazak, A. E., Rash-Ellis, D. L., ... Deatrick, J. A. (2020). Family resilience from the perspective of caregivers of youth with sickle cell disease. Journal of Pediatric Hematology \& Oncology, 42, 100-106. https://doi.org/10.1097/mph.0000000000001682.

Schoenthaler, A., Rosenthal, D. M., Butler, M., \& Jacobowitz, L. (2018). Medication adherence improvement similar for shared decision-making preference or longer patient-provider relationship. The Journal of the American Board of Family Medicine, 31, 752-760. https://doi.org/10.3122/jabfm.2018.05.180009.

Steinberg, D. M., Schneider, N. M., Guler, J., Garcia, A. M., Kullgren, K. A., Agoston, A. M., ... Judd-Glossy, L. (2020). Pediatric consultation liaison psychology services during the COVID-19 pandemic: pivoting to provide care. Clinical Practice in Pediatric Psychology.

Talevi, D., Socci, V., Carai, M., Carnaghi, G., Faleri, S., Trebbi, E., ... Pacitti, F. (2020). Mental health outcomes of the CoViD19 pandemic. Rivista di Psychiatria, 55, 137-144. https://doi. org/10.1708/3382.33569.

Tillery, R., Howard Sharp, K. M., Okado, Y., Long, A., \& Phipps, S. (2016). Profiles of resilience and growth in youth with cancer and healthy comparisons. Journal of Pediatric Psychology, 41, 290-297. https://doi.org/10.1093/jpepsy/jsv091.

Weaver, M. S., \& Wiener, L. (2020). Applying palliative care principles to communicate with children about COVID-19. Journal of Pain and Symptom Management, 60, e8-e11. https://doi.org/10.1016/j. jpainsymman.2020.03.020.

Xiang, M., Zhang, Z., \& Kuwahara, K. (2020). Impact of COVID-19 pandemic on children and adolescents' lifestyle behavior larger than expected. Progress in Cardiovascular Diseases. https://doi. org/10.1016/j.pcad.2020.04.013.

Zachariah, P., Johnson, C. L., Halabi, K. C., Ahn, D., Sen, A. I., Fischer, A., ... Saiman, L. (2020). Epidemiology, clinical features, and disease severity in patients with coronavirus disease 2019 (COVID-19) in a Children's Hospital in New York City, New York. Journal of the American Medical Association Pedaitrics, e202430. https://doi.org/10.1001/jamapediatrics.2020.2430.

Publisher's Note Springer Nature remains neutral with regard to jurisdictional claims in published maps and institutional affiliations. 